University of Louisville

ThinkIR: The University of Louisville's Institutional Repository

Faculty Scholarship

$5-2008$

\title{
A longitudinal study of cortical EEG activity in adolescents.
}

\author{
Patrick Pössel \\ University of Louisville \\ Hanna Lo \\ University of Tuebingen \\ Anna Fritz \\ University of Tuebingen \\ Simone Seemann \\ University of Tuebingen
}

Follow this and additional works at: https://ir.library.louisville.edu/faculty

Part of the Counseling Psychology Commons

\section{Original Publication Information}

Pössel, Patrick, Hanna Lo, Anne Fritz and Simone Seemann. "A Longitudinal Study of Cortical EEG Activity in Adolescents." 2008. Biological Psychology 78(2): 173-178.

This Article is brought to you for free and open access by ThinkIR: The University of Louisville's Institutional Repository. It has been accepted for inclusion in Faculty Scholarship by an authorized administrator of ThinkIR: The University of Louisville's Institutional Repository. For more information, please contact thinkir@louisville.edu. 
Running head: CORTICAL EEG ACTIVITY IN ADOLESCENTS

\author{
A Longitudinal Study of Cortical EEG Activity in Adolescents ${ }^{1}$ \\ Patrick Pössel, Hanna Lo, Anne Fritz, and Simone Seemann \\ University of Tübingen, Germany
}

Correspondence and requests for reprints should be addressed to

Prof. Dr. Patrick Pössel

Department of Educational and Counseling Psychology

College of Education \& Human Development

University of Louisville

Louisville, KY 40292

Phone: 502-852-0623

Fax: 502-852-0629

e-mail: Patrick.possel@ louisville.edu

${ }^{1}$ The first author is at the Department of Educational and Counseling Psychology, University of Louisville, now. 


\begin{abstract}
Background: The objective of this study is to test Davidson's, and Heller and Nitschke's models stating cortical activity in adolescents to be a marker for increased risk for depression.

Methods: Alpha activity was measured in 80 adolescents from medial-frontal (F3/4), lateralfrontal (F7/8), and medial-parietal (P3/4) electrodes, as well as self-reported depression and anxiety twice within 12 months. Stepwise hierarchical regression analyses with anxiety as covariate were calculated with alpha asymmetry as predicting variable and depression as target variable and vice versa.

Results: Independent of whether anxiety was used as covariate or not, frontal and parietal alpha asymmetry predict depression, but depression did not predict alpha activity.

Conclusions: The results partially support both models and conclusions drawn by studies with remitted depressed subjects. However, it remains unclear if anxious arousal has a partial influence on the predictive association between alpha activity and depression.
\end{abstract}

Keywords: alpha activity; adolescents; depressive symptoms; anxiety symptoms; longitudinal study. 


\section{Introduction}

Davidson $(1995,1998$ a) proposed a model regarding the regulation of anterior asymmetry and emotion. The model predicts that approach-related positive emotions (e.g., pride, enthusiasm) are linked to hyperactivity of the left frontal hemisphere while withdrawal-related negative emotions (e.g., fear, disgust) are associated with a relative hyperactivity of the right frontal hemisphere. With regard to depression, Davidson (1998b) suggested that individual differences in prefrontal asymmetry are most appropriately viewed as diathesis that bias a person's affective style, and then in turn modulate an individual's vulnerability to develop depression. When an individual with left-sided prefrontal hypoactivation is exposed to negative life events over a prolonged period of time, the model predicts an increase in the probability of developing depression.

This model is supported by several studies (for a review see Davidson, 2004). Gotlib et al. (1998), for example, confirmed the expected left frontal hypoactivity in acute depressed as well as remitted patients, and a left frontal hyperactivity in normal controls. Moreover, they were able to show that acute and remitted depressed patients do not differ significantly with regard to frontal asymmetry. These results are consistent with Davidson's hypothesis (1998b) of prefrontal asymmetry being a diathesis of depression that may have preceded the first episode and therefore serves as an endophenotype of risk for depression (Allen et al., 2004a). It cannot be ruled out, however, that activity results in remitted subjects merely reflect the effects of past depressive episodes, for remitted depressed people show generally more depressive symptoms than normal controls. Furthermore, it could be assumed that an episode of major depression changes cortical activity even after remission, which is known as "scar hypothesis". Bruder et al. (2005) found some support for the scar hypothesis in their longitudinal study with adult offspring of depressed and nondepressed parents, as offspring of depressed and nondepressed parents did not differ at frontal electrodes, but in parietal alpha activity. Moreover, frontal activity was different between 
subjects with and without life-time major depression, independent of the health status of their parents. Thus, it is possible that differences between both groups are caused by this unequal intensity of symptoms as opposed to an increased risk of remitted depressed persons for future depressive episodes. Based on these findings, longitudinal research with at least two time points including subjects without life-time major depression at the first time point is needed in order to decide if left frontal hypoactivity is really a marker for increased risk for depression. If left frontal hypoactivation is a stable marker for increased risk for depression, alpha activity in the left frontal cortex at one time point should predict depression at a later time point.

Despite the findings mentioned above, empirical support for Davidson's model $(1995,1998 \mathrm{a})$ in adolescents is limited and contradictory. For example, Graae et al. (1996) showed that adolescent females, who attempted suicide and most of whom met criteria for major depression, to differ in asymmetry from controls in the expected direction, but the differences in cortical activity were more related to their suicidal intentions rather than to the severity of depressive symptoms. Tomarken et al. (2004) compared adolescents of mothers with a history of depression with those, whose mothers showed no lifetime axis I psychopathology. The authors described the expected left frontal hypoactivity in high risk adolescents but the socio-economic status contributed more variance to the prediction of frontal brain asymmetry than mothers' psychopathology. Finally, Ehlers et al. (2001) showed correlations between alpha asymmetry and depressed behavior in a sample of Native American youths in various reservations.

High comorbidity of depression and anxiety disorders was proposed as a possible cause for contradictory results (Heller et al., 1997; Bruder et al., 1997; Heller \& Nitschke, 1998; Kentgen et al., 2000), which may be especially important for research in adolescents because of a very high comorbidity rate in this age (Angold et al., 1999). Davidson's model predicts right frontal hyperactivity and increased frontal asymmetry in subjects with comorbid depression and anxiety 
disorder compared to subjects with the sole diagnosis of depression (Davidson, 1995, 1998a). Heller et al. (1997), however, postulated, beside a left frontal and right parietal hypoactivation in depression, two subtypes of anxiety, which are associated with different patterns of cortical activity: Anxious arousal (e. g., physiological hyperarousal and somatic tension) is associated with right parietal hyperactivity, whereas anxious apprehension (e. g., rumination and worry) is associated with left frontal hyperactivity. Thus, a right parietal hypoactivity due to depression can be superimposed by an additive effect of right parietal hyperactivity due to anxious arousal. Furthermore, contrary patterns of frontal activity in depression and anxious apprehension could reduce the expected asymmetry between left and right frontal activity in depressed adolescents. Summarized, comorbid anxiety can decrease measurable asymmetry in depressed youths between left and right hemisphere.

Support for this hypothesis is offered by the studies of Bruder et al. (1997) and Kentgen et al. (2000). Bruder et al. (1997) compared left and right parietal regions and showed depressive patients without an anxiety disorder to have less right parietal activity compared to depressive patients with an additional anxiety disorder. Kentgen et al. (2000) compared depressed female adolescents with and without anxiety disorder and showed that only those without anxiety have less right parietal activity. Both studies did not find differences in frontal regions. These studies point to the potential importance of comorbidity of depression and anxiety and its consequence on electrophysiological activity in the cortex. Due to these findings, it appears necessary to use present anxiety symptoms as covariates in statistical analyses in order to investigate the relationship of depression and alpha activity in adolescents.

Based on the literature, the empirical evidence that alpha asymmetry may be a stable marker for increased risk for depression especially in adolescents is limited. In order to test the hypothesis that left frontal hypoactivation is a stable marker for increased risk for depression in 
adolescents and not a result of past depressive episodes, longitudinal research with mentally healthy youth at the first time point accounting for variance of anxiety on alpha asymmetry is needed. Based on the models of Davidson (1995, 1998a) as well as Heller and Nitschke (1998), the following hypotheses were deducted: It is expected that left frontal hypoactivation predicts depression scores at a later time point. Furthermore, as both models do not posit depression to be a risk factor for a changed alpha activity, depression at the first time point should not predict later alpha activity. In line with the model of Heller and Nitschke (1998), a right parietal hypoactivity should predict higher depression after accounting for variance of anxiety while depression should not predict later right parietal hypoactivity after accounting for variance of anxiety. Without controlling for anxiety, no relationship between right parietal hypoactivity and depression should be detectable.

Methods and Materials

\section{$\underline{\text { Participants }}$}

A total of 100 mentally healthy adolescents from the area of Tuebingen (southwest of Germany) at the age of 13 to 15 years participated in the study between August 2002 and May 2004. Youth with a life-time diagnosis of life-time depression were excluded to rule out that correlations between alpha activity and depression scores reflect the effects of past depressive episodes. Ten left-handed students measured by the Edinburgh Inventory (Oldfield, 1971) were excluded (cf. Schaffer et al., 1983). In order to participate adolescents as well as their legal guardians filled in a written consent form. They received information about EEG measurements in general, about the procedure, as well as about the aim of the study, i.e., to test possible associations between moods and EEG. Participants could leave the experiment any time without giving reasons. The study was approved by the Institutional Review Board of the German 
Psychological Association. Participation was compensated by $€ 13$ for each of two lab sessions. Of all participating students, one subject showed asymmetry scores of more than four standard deviations from the mean and was subsequently excluded from the sample. Five participants were not considered in the electrophysiological analysis due to strong artifacts in resting EEG. Four adolescents did not participate on the second measurement 12 months after their first participation. The remaining 80 adolescents comprised 35 females and 45 males with a mean age of 13.92 years $(\mathrm{SD}=.57)$.

\section{$\underline{\text { Instruments }}$}

\section{Depressions-Screening Questionnaire (DSQ)}

Wittchen and Perkonigg (1997) developed the Depressions-Screening Questionnaire (DSQ) as an instrument to measure major depression. The DSQ consists of 12 questions to assess the 9 symptoms of depression based on DSM-IV criteria and formulations (American Psychiatric Association, 1994). The adolescents were asked to answer the questions with regard to all past experiences using a three-point Likert scale (no - sometimes - most days). Consistent with the DSM-IV, the diagnosis of major depression was given if an adolescent rated at lease five items as experienced at "most days" in the same two weeks or more. Subjects with a diagnosis of major depression were excluded from this study. Test-retest reliability (Kappa) varied from .68 - .92 on both the symptom level and the diagnostic level, which we considered satisfactory. Validity by comparison with a DIA-X (German CIDI version) diagnosis of major depression was .76.

\section{$\underline{\text { Self-Rating Questionnaire for Depressive Disorders (SBB-DES) }}$}

The Self-Rating Questionnaire for Depressive Disorders (SBB-DES) developed by Döpfner and Lehmkuhl (2000) measures symptom criteria in accordance with DSM-IV diagnoses of depressive disorders (APA, 1994). The 29 items are combined according to the four symptom groups in DSM-IV and ICD-10 (WHO, 2000): depressive symptoms, somatic syndrome (ICD- 
10), dysthymia (ICD-10), and dysthymic disorder (DSM-IV). Additionally, a total score for the depression scale can be calculated, which was used in our analyses. Each item was rated for severity of the symptom. The self-rating questionnaire is applicable to adolescents of average intelligence from age eleven. Cronbach's $\alpha$ of the total scale of severity was .92 .

\section{Self-Rating Questionnaire for Anxiety Disorders (SBB-ANG)}

The Self-Rating Questionnaire for Anxiety Disorders (SBB-ANG) developed by Döpfner and Lehmkuhl (2000) uses 31 items to measure symptom criteria for the diagnosis of anxiety disorders according to DSM-IV (APA, 1994) in children and adolescents starting at age eleven. The items are categorized according to the four symptom groups noted in the DSM-IV and the ICD-10: separation anxiety, generalized anxiety, social anxiety, and phobic anxiety. Response categories and test scores are analogous to the SBB-DES. For our analysis we used a total score for anxiety. Cronbach's $\alpha$ of the total scale of severity was .90 .

\section{$\underline{\text { Procedure }}$}

After the lab and especially the EEG equipment was shown to the participants and they filled in the questionnaires, the EEG was recorded in an electrically shielded booth. Participants were seated in an armchair in an upright position. They were instructed to remain silent and as relaxed as possible and to try to inhibit movements of any kind. The EEG was recorded during four 2min periods: two eyes-open trials $(\mathrm{O})$ and two eyes-closed trials $(\mathrm{C})$. Two randomly assigned, counterbalanced orders were used (C-O-C-O and O-C-O-C). Participants were informed about the recording condition in advance by the experimenter. In order to minimize the influence of eye movements, subjects in the eyes-open condition were instructed to fixate on a cross, presented on a monitor at approximately $100 \mathrm{~cm}$ distance.

After 12 months the adolescents participated in the same measurements following the same procedure. 


\section{Electrophysiological Recording and Analysis}

Spontaneous EEG was recorded at medial-frontal (F3/F4), lateral-frontal (F7/F8), and medialparietal (P3/P4) electrode sites following the 10-20 International System (Jasper, 1958) with a nose reference. This reference was chosen because it is centrally located, relatively inactive (e.g., compared to $\mathrm{Cz}$ ), and can be easily applied. It is used in many studies that find the expected alpha asymmetry (e.g., Bruder et al., 1997; Kentgen et al., 2000; Papousek \& Schulter, 2002). Furthermore, a meta-analysis about Davidson's model describes the highest effect sizes for the nose reference compared to $\mathrm{Cz}$, mastoid $(\mathrm{A} 1+\mathrm{A} 2)$ and average references (Jakobi, 2005). Ag$\mathrm{AgCl}$ ring electrodes were used, with impedances below $5 \mathrm{k} \Omega$. For continuous recording, we used a 32-channel amplifier (SYNAMPS) and the acquisition software Acquire 4.0 (Neuroscan). EEGs were recoded at a gain of $10 \mathrm{k} \Omega$ (5 for eye channels) with a bandpass of $0.53-40 \mathrm{~Hz}$ and a $50 \mathrm{~Hz}$ notch filter to prevent artifacts from alternating current network. The data were digitized using an analog digital converter at $250 \mathrm{~Hz}$ and saved on a hard disk.

The computation of EEG data was conducted with the analysis software Vision Analyser (Brain Products). Each of the four 2-min-baseline measurements was segmented into 231 epochs of $2.05 \mathrm{~s}$ in duration, overlapped by $75 \%$ (cf. Tomarken et al., 1992). Artifacts were removed automatically, i.e. epochs with an amplitude $>50 \mu \mathrm{V}$ or $<-50 \mu \mathrm{V}$ on each channel (cf. Reid et al., 1998). All artifact-free epochs were extracted through a Hamming window (10\%).

Subsequently, a Fast Fourier Transform (FFT) was used to derive estimates of spectral power. At each electrode, alpha power was calculated by averaging the alpha band $(8-13 \mathrm{~Hz})$ power values of all artifact-free epochs. To normalize the data, all alpha power values were log-transformed using the natural logarithm. Then aggregated alpha power values were provided by averaging the scores of all four baselines and weighting each mean by the number of artifact-free segments. The conditions "eyes open" and "eyes closed" were collapsed because alpha power was reduced 
in the eyes open condition compared to the eyes closed condition $(F(3,84)=72.16, p<.001)$, but independent of the other factors (depression score, gender, hemisphere). Finally, asymmetry scores were calculated (right - left) as primary outcome variables.

Although it would have been desirable to use absolute power as outcome variable due to its ability to determine which hemisphere is responsible for differences that might exist in alpha activity (Thibodeau et al., 2006), absolute power was not used in this study because of a high correlation between left and right absolute power. This correlation causes a set of nearly equal but opposite beta weights for corresponding electrode sites (Allen et al., 2004b).

\section{$\underline{\text { Data Analysis }}$}

Stepwise hierarchical regression analyses were calculated using the software package SPSS 11.5. To test the influence of cortical alpha activity on self-reported depression 12 months later a hierarchical regression analysis with SBB-DES score at the first time point and anxiety score at the first and second time point were entered at step 1 as covariates. At step 2, the EEG asymmetry values at $\mathrm{F} 3 / 4, \mathrm{~F} 7 / 8$, and $\mathrm{P} 3 / 4$ to the first time point were entered as predictor variables in this analysis. The depression score at the second time point was used as target variable.

To test the influence of self-reported depression on cortical alpha activity 12 months later, alpha activity at the first time point and anxiety score at the first and second time point were entered at step 1 in another regression analysis as covariates. At step 2, the SBB-DES score to the first time point was entered as predictor variable. As target variables EEG asymmetry values at F3/4, F7/8, and P3/4 to the second time point were used.

The regression analyses were recalculated without anxiety as covariate. With a sample size of $n=80$ and $\alpha$ set at .05 the power in these analyses were adequate $(>.80)$ to detect medium or larger effect sizes (Cohen, 1998). 
Results

$\underline{\text { Preliminary Results }}$

Depression $(\mathrm{M}=.67, \mathrm{SD}=.59)$ and anxiety scores $(\mathrm{M}=.67, \mathrm{SD}=.60)$ at the first time point $(\mathrm{r}=.92, \mathrm{p}<.001)$ as well as at the second time point $(\mathrm{SBB}-\mathrm{DES}: \mathrm{M}=.66, \mathrm{SD}=.57, \mathrm{SBB}-\mathrm{ANG}$ $=.63, \mathrm{SD}=.55 ; \mathrm{r}=.82, \mathrm{p}<.001)$ were significantly correlated indicating high comorbidity of depression and anxiety symptoms in the sample. While the SBB-DES and SBB-ANG scores for the whole group were relatively stable over the course of the study, $7.1 \%(n=6)$ of the adolescents had at least one episode of major depression in the 12 months between both time points. The bivariate distributions of alpha asymmetry at electrode sites at first time point versus depression severity at second time point are shown in figure 1.

The retest-reliabilities of the asymmetry values over 12 months at the electrode sizes were $\mathrm{r}=$ .23 through .47 . These relatively low retest-reliabilities for asymmetry values are consistent with the literature (e.g., Tomarken et al., 1992).

\section{Alpha Activity predicting Depression}

In line with the expectation, alpha asymmetry at the medial-frontal (F3/4) and medial-parietal (P3/4) electrodes predicted depression 12 months later while the alpha activity at lateral-frontal (F7/8) electrodes did not significantly predict depression (Table 1), independent of whether anxiety was used as covariate $(\mathrm{F}(3,68)=4.52, \mathrm{p}=.006)$ or not $(\mathrm{F}(3,70)=6.19, \mathrm{p}=.001)$.

\section{Depression predicting Alpha Activity}

Again in line with the expectation, depression at the first time point did not predict alpha asymmetry 12 months later when using EEG alpha activity at the first time point as covariate. These results are independent of whether anxiety is used as covariate $(F 3 / 4: F(1,68)=2.35, p=$ 
$.130 ; \mathrm{F} 7 / 8: \mathrm{F}(1,68)=.28, \mathrm{p}=.602 ; \mathrm{P} 3 / 4: \mathrm{F}(1,68)=.30, \mathrm{p}=.588)$ or not $(\mathrm{F} 3 / 4: \mathrm{F}(1,71)=.40, \mathrm{p}=$ $.532 ; \mathrm{F} 7 / 8: \mathrm{F}(1,71)=.13, \mathrm{p}=.721 ; \mathrm{P} 3 / 4: \mathrm{F}(1,71)=.11, \mathrm{p}=.741)$.

\section{Discussion}

The goal of this study was to test the connections between EEG alpha activity and depression in adolescents in a longitudinal study. Davidson $(1995,1998$ a) postulated that left hemisphere hypoactivity predicts depression to a later time point while no predictive relationship between depression and later frontal activity was expected. Heller et al. (1997), however, postulated a left frontal and right parietal hypoactivity in depression. Furthermore, they posited anxious arousal to be associated with right parietal hyperactivity, whereas anxious apprehension is associated with left frontal hyperactivity. Based on these contrary patterns of cortical activity, the left frontal hypoactivity due to depression can be expected to be superimposed by comorbid anxious apprehension while the depressive right parietal hypoactivity can be reduced by comorbid anxious arousal. Summarized, comorbid anxiety could decrease measurable asymmetry in depressed youths between left and right hemisphere. The fact that alpha activity predicts selfreported depression 12 months later in our study with adolescents supports the hypothesis that alpha activity is a marker for an increased risk for depressive disorders (Davidson, 1995). Furthermore, depressive symptoms do not predict alpha activity 12 months later.

Constistent with Davidson's model, we found left frontal hypoactivity to predict depression. However, in our study left parietal hypoactivity predicted depression as well. In one of their studies, Davidson et al. (1995) showed left hemisphere hypoactivity to be connected with melancholic depression while right hemisphere hyperactivity is connected with non-melancholic depression. Therefore, it can be hypothesized that our frontal and parietal findings base on existence of melancholic and non-melancholic depression in the sample, especially since anxiety 
as a predictor for this left hemisphere hypoactivity can be excluded as it was used as covariate in the analyses. Both studies are also in accordance with Heller and Nitschke's model (1998) that states a relatively lower left frontal and a higher right parietal activity to predict depression.

At first view the fact that a) using anxiety as covariate did not have an impact on or altered the relationship between alpha activity and depression and b) left hypoactivity and right hyperactivity in parietal regions predicted depression, seems not to support the models of Davidson (1995, 1998a) as well as Heller and Nitschke (1998). However, both parts of our results can be explained by the same mechanism. Studies of Nitschke et al. (1999) and Davidson et al. (2000) showed that mainly anxious arousal and anxiety in a fearful situation, respectively, is associated with alpha activity while anxious apprehension shows no correlation to alpha activity. Furthermore, although some of the anxiety disorders measured by the SBB-ANG might have a strong anxious arousal part, most questions of the SBB-ANG focus on anxious apprehension (e.g., separation anxiety: "I worry that something bad could happen to my parents."; social anxiety: "I worry a lot whether I behave in the correct way in situations with strangers."). If so, we could only account for variance of anxious apprehension that is low or may be not associated with cortical alpha activity while accounting for variance of anxious arousal was not possible. Combined with a high correlation between depression and anxiety in our sample (first measurement: $r=.82$; measurement 12 months later: $r=.92)$, the lack of accounting for variance of anxious arousal might be the explanation why parietal asymmetry seems to predict depression. All together, it could be that parietal alpha activity does not predict depression, but anxious arousal does, which might be correlated with depression. This could lead to the overestimation of the association between alpha activity and depression 12 months later. To test this hypothesis, another longitudinal study is needed with instruments that can separate depression, anxious apprehension, and anxious arousal. 
Using the SBB-DES and SBB-ANG to measure depression and anxiety can be seen as first methodological limitation of the presented study for different reasons. First, these instruments are not able to differ between depression, anxious arousal, and anxious apprehension. Therefore, the Mood and Anxiety Symptom Questionnaire-Children (MASQ-C, Rudolph, 2003) and the adaptation of the Penn State Worry Questionnaire (PSWQ) should be used in replication studies, for these questionnaires can differentiate between these variables (Chorpita et al., 1997). Second, this study integrated anxiety symptoms in adolescents only as covariate in step-wise regression analyses instead of composing subgroups with and without anxiety symptoms. However, a subsequent division of adolescents in subgroups was not possible due to the high correlation between depression and anxiety, which is very common during adolescence (Angold et al., 1999). Third, the selection of subjects only relied on self-rating scales, not on clinical diagnoses. Although this procedure has been used successfully in several studies (Reid et al., 1998; Schaffer et al., 1983), a replication should prefer a clinical interview over self-rating. Finally, frontal EEG activity showed only moderately stability. As Davidson (1998b) suggests the use of multisession measures to better estimate an individual's true alpha power, replication studies should measure alpha activity in adolescents over multiple time points and choose only those subjects for further analyses that are found to have stable alpha activity patterns.

Besides these limitations some of the advantages of the presented study shall be mentioned as well. The inclusion of a relatively large sample size can be seen as one of the main advantages of our study. While the majority of studies using alpha activity have less than thirty participants, the presented study has a sample size of $n=80$, which allows for the detection of medium effect sizes. Another advantage is the application of a 12 months longitudinal design. As far as we know, this is the first longitudinal study in adolescents testing the hypothesis whether cortical alpha activity is a marker for an increased risk for depression. 
In sum, frontal and parietal EEG asymmetry predict depression 12 months later in adolescents 13 to 15 years of age. These results partially support the models of Davidson $(1995,1998$ a) as well as Heller and Nitschke (1998). Depression does not predict alpha activity 12 months later, what supports the conclusions drawn by studies with remitted depressed subjects. However, it remains unclear if anxious arousal has a partial influence on the predictive association between alpha activity and depression. 


\section{References}

Allen, J.B., Coan, J.A., Nazarian, M. 2004b. Issues and assumptions on the road from raw signals to metrics of frontal EEG asymmetry in emotion. Biological Psychology 67, 183-218.

Allen, J.B., Urry, H.L., Hitt, S.K., Coan, J.A., 2004a. The stability of resting frontal electroencephalographic asymmetry in depression. Psychophysiology 41, 269-280.

American Psychiatric Association, 1994. Diagnostic and Statistical Manual of Mental Disorders, fourth ed. American Psychiatric Press, Washington, D. C..

Angold, A., Costello, E.J., Erkanli, A., 1999. Comorbidity. Journal of Child Psychology \& Psychiatry 40, 57-87.

Bruder, G.E., Fong, R., Tenke, C.E., Leite, P., Towey, J.P., Stewart, J.E. et al., 1997. Regional brain asymmetries in major depression with or without an anxiety disorder: A quantitative electroencephalographic study. Biological Psychiatry 41, 939-948.

Bruder, G.E., Tenke, C.E., Warner, V., Nomura, Y., Grillon, Ch., Hille, J., Leite, P., Weissman, M.M., 2005. Eletroencephalographic measures of regional hemispheric activity in offspring at risk for depressive disorders. Biological Psychology 57, 328-335.

Chorpita, B.F., Tracey, S.A., Brown, T.A., Collica, T.J., Barlow, D.H., 1997. Assessment of worry in children and adolescents: An adaptation of the Penn State Worry Questionnaire. Behaviour Research and Therapy 35, 569-581.

Cohen, J., 1998. Statistical power analysis for the behavioral sciences. Lawrence Erlbaum Associates, Hillside, NJ.

Cohen, J., Cohen, P., 1983. Applied multiple regression/correlation analysis for the behavioral sciences, second ed. Erlbaum, Hillsdale, NJ. 
Davidson, R.J., 1995. Cerebral asymmetry, emotion and affective style, in: Davidson, R.J., Hugdahl, K. (Eds.), Brain Asymmetry. MIT Press, Cambridge, MA, pp. 361-387.

Davidson, R.J., 1998a. Affective style and affective disorders: Perspectives from affective neuroscience. Cognition and Emotion 12, 307-330.

Davidson, R.J., 1998b. Anterior electrophysiological asymmetries, emotion, and depression: Conceptual and methodological conundrums. Psychophysiology 35, 607-614.

Davidson, R.J., 2004. What does the prefrontal cotex "do" in affect: perspectives on frontal EEG asymmetry research. Biological Psychology 67, 219-233.

Davidson, R.J., Larson, C., Abercrombie, H., 1995, October. Prefrontal electrophysiological asymmetries differentiate between melancholic and non-melancholic depression. Annual Meeting of the Society for Research in Psychopathology, Iowa City, IO.

Davidson, R.J., Marshall, J.R., Tomarken, A.J., Henriques, J.B., 2000. While a phobic waits: regional brain electrical and autonomic activity in social phobics during anticipation of public speaking. Biological Psychiatry 47, 85-89.

Döpfner, M., Lehmkuhl, G., 2000. DISYPS-KJ: Diagnostik-System für psychische Störungen im Kindes- und Jugendalter nach ICD-10 und DSM IV. Verlag Hans Huber, Bern, Switzerland.

Ehlers, C.L., Wall, T.L., Garcia-Andrade, C., Phillips, E., 2001. EEG asymmetry: Relationship to mood and risk for alcoholism in Mission Indian youth. Biological Psychiatry 50, 129-136.

Gotlib, I.H., Ranganath, C., Rosenfeld, J.P., 1998. Frontal EEG alpha asymmetry, depression, and cognitive functioning. Cognition and Emotion 12, 449-478. 
Graae, F., Tenke, C., Bruder, G., Rotheram, M.J., Piacentini, J., Castro-Blanco, D., et al., 1996. Abnormality of EEG alpha asymmetry in female adolescent suicide attempters. Biological Psychiatry 40, 706-713.

Heller, W., Nitschke, J.B., 1998. The puzzle of regional brain activity in depression and anxiety: The importance of subtypes and comorbidity. Cognition and Emotion 12, 421-447.

Heller, W., Nitschke, J.B., Etienne, M.A., Miller, G.A., 1997. Patterns of regional brain activity differentiate types of anxiety. Journal of Abnormal Psychology 106, 376-385.

Jakobi, U., unpublished. A meta-analysis about EEG-alpha-asymmetries and depression. Diploma thesis. University of Tuebingen, Department of Psychology.

Jasper, H.H., 1958. The ten twenty electrode system of the International Federation. Electroencephalography and Clinical Neurophysiology 10, 371-375.

Kentgen, L.M., Tenke, C.E., Pine, D.S., Fong, R., Klein, R.G., Bruder, G.E, 2000. Electroencephalographic asymmetries in adolescents with major depression: Influence of comorbidity with anxiety disorders. Journal of Abnormal Psychology 109, 797-802.

Nitschke, J.B., Heller, W., Palmieri, P.A., Miller, G.A., 1999. Contrasting patterns of brain activity in anxious apprehension and anxious arousal. Psychophysiology 36, 628-637.

Oldfield, R.C., 1971. The assessment and analysis of handedness: The Edinburgh Inventory. Neuropsychologia 9, 97-113.

Papousek, I., Schulter, G., 2002. Covariations of EEG asymmetries and emotional states indicate that activity at frontopolar locations is particularly affected by state factors. Psychophysiology $39,350-360$. 
Reid, S.A., Duke, L.M., Allen, J.J.B., 1998. Resting frontal electroencephalographic asymmetry in depression: Inconsistencies suggest the need to identify mediating factors. Psychophysiology 35, 389-404.

Rudolph, K., unpublished. Mood and Anxiety Symptom Questionnaire - Children. University of Illinois, Urbana-Champaign, Illinois, USA.

Schaffer, C.E., Davidson, R.J., Saron, C., 1983. Frontal and parietal electroencephalogram asymmetry in depressed and non-depressed subjects. Biological Psychiatry 18, 753-762.

Thibodeau, R, Jorgensen, RS, Kim, S, 2006. Depression, anxiety, and resting frontal EEG asymmetry: a meta-analytic review. Journal of Abnormal Psychology 115, 715-729.

Tomarken, A.J., Davidson, R.J., Wheeler, R.E., Kinney, L., 1992. Psychometric properties of resting anterior EEG asymmetry: Temporal stability and internal consistency. Psychophysiology 29, 576-592.

Tomarken, A.J., Dichter, G.S., Garber, J., Simien, Ch., 2004. Resting frontal brain activity: linkage to maternal depression and socio-economic status among adolescents. Biological Psychology 67, 77-102.

Wittchen, H.U., Perkonigg, A., 1997. DIA-X Fragebögen, in: Wittchen, H.U., Pfister, H. (Eds.), Instruktionsmanual zur Durchführung von DIA-X Interviews. Swets Test Services, Frankfurt, pp. 11-20.

World Health Organization, 2000. The ICD-10 Classification of Mental and Behavioural Disorders: Clinical Descriptions and Diagnostic Guidelines, fourth ed. World Health Organization, Geneva, Switzerland. 
Table 1

Results of the regression analysis with alpha asymmetry as predictor and depression as target variable

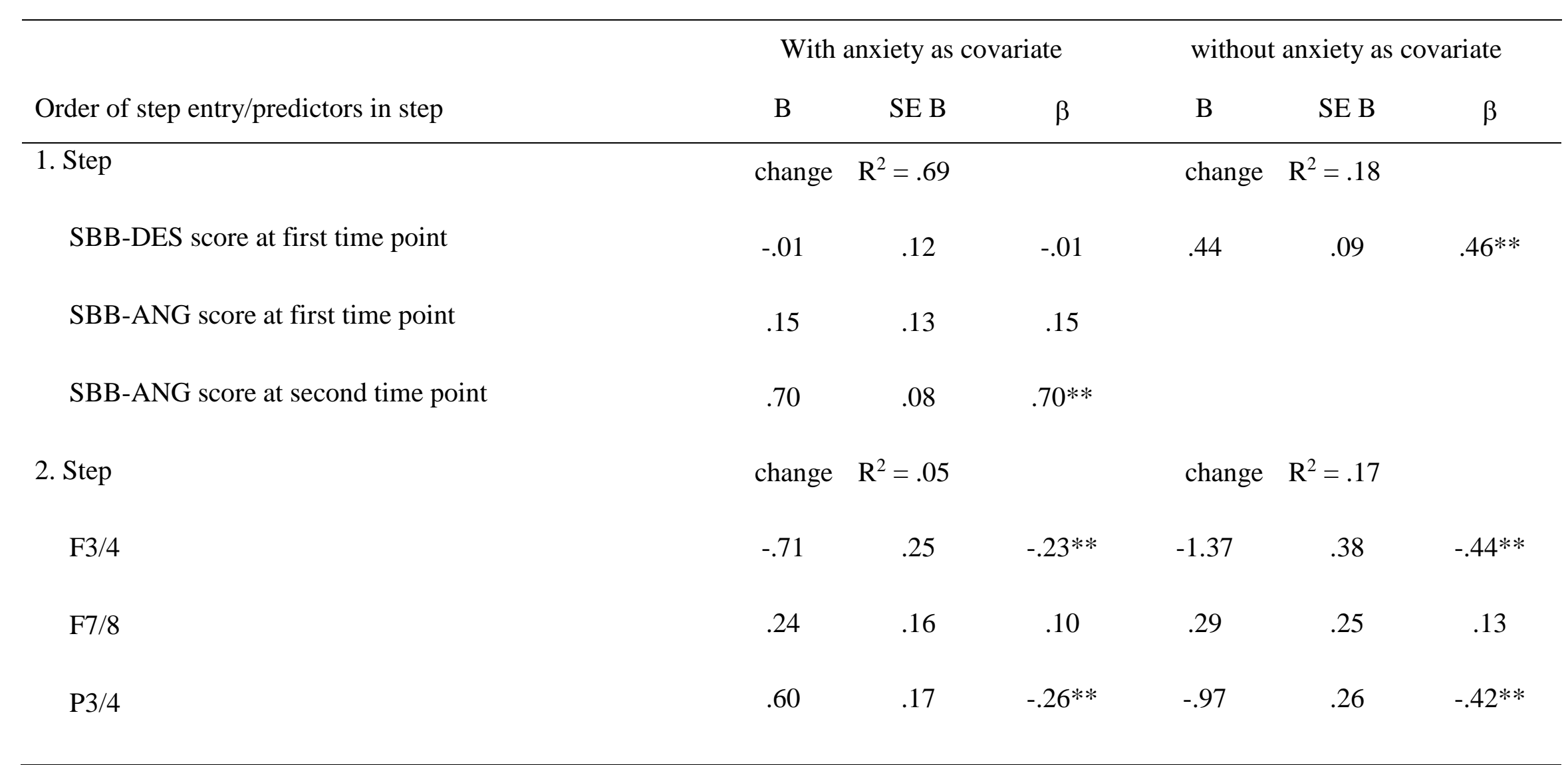

Footnote: $\mathrm{F} 3 / 4=(\ln \mathrm{F} 4-\ln \mathrm{F} 3) ; \mathrm{F} 7 / 8=(\ln \mathrm{F} 8-\ln \mathrm{F} 7) ; \mathrm{P} 3 / 4=(\ln \mathrm{P} 4-\ln \mathrm{P} 3){ }^{*}=p \leq .05 ; * *=p \leq .01$ 
Figure 1

Bivariate distributions of alpha asymmetry at electrode sites at the first time point and depression severity at the second time point 

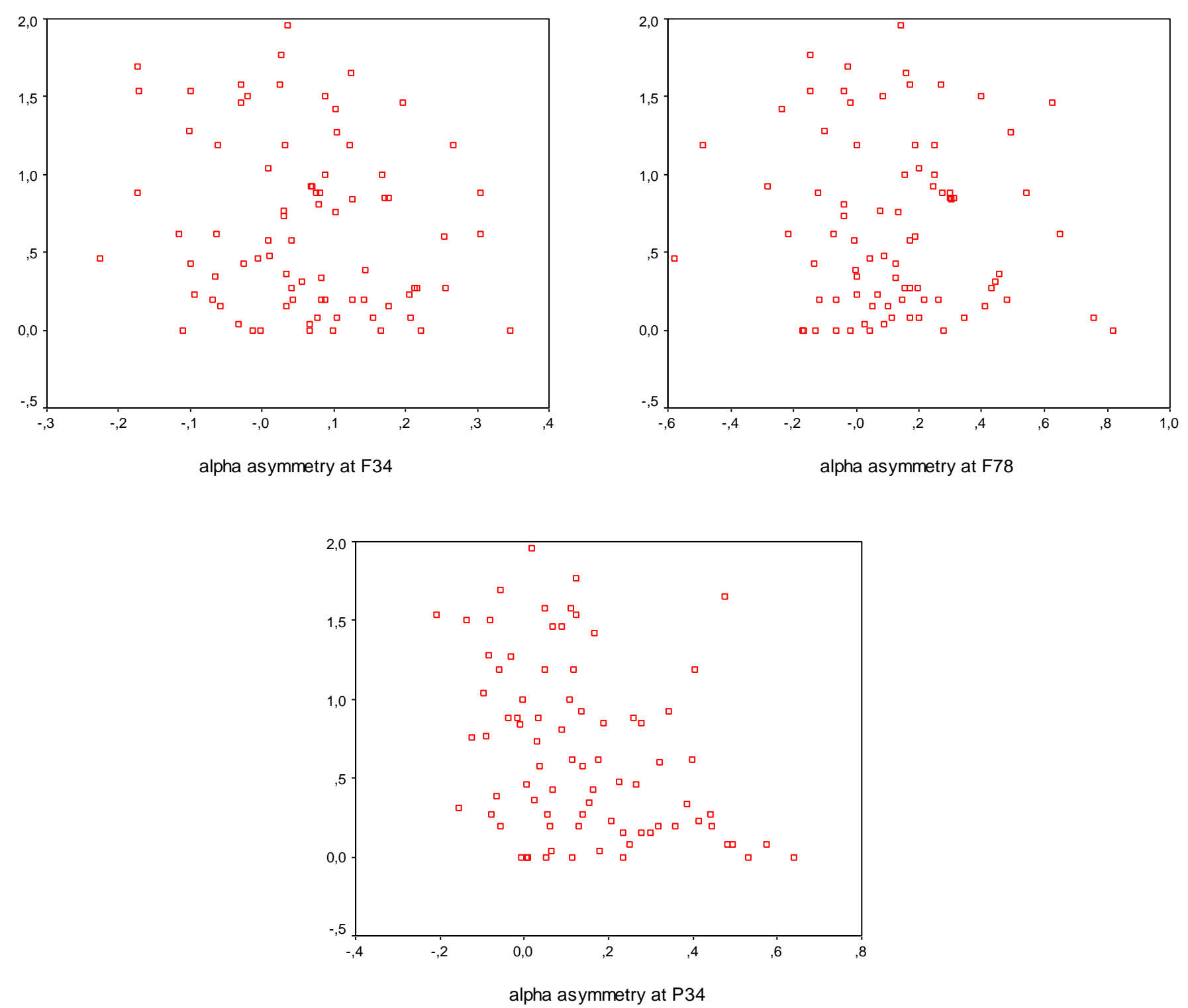


\section{Acknowledgement}

We would like to thank the adolescents, parents, and teachers involved in this project and express our gratitude to the local education agency of Tübingen for making this project possible. Furthermore, we are grateful to three anonymous reviewers and the Associate Editor Dr. Allen for their extensive and valuable comments on an earlier version of this article.

The project was supported by the grant PO 766/1-1 from the Deutsche Forschungsgemeinschaft (German Research Foundation) to Patrick Pössel. 